\title{
Secondary Epiretinal Membrane After Trabeculectomy
}

\author{
Luísa Vieira, MD,* Ana Cabugueira, MD,* Bárbara Borges, MD,* \\ Vanessa Carvalho, MD,* Manuel Noronha, MD, ${ }^{*}$ Luis Abegão Pinto, MD, PhD, ${ }^{*}+$ \\ Maria Reina, MD,* and Marco Dutra Medeiros, MD* ${ }_{*}^{*}$
}

\begin{abstract}
Purpose: To determine the frequency of epiretinal membranes $(E R M)$ in eyes with primary open-angle glaucoma subjected to trabeculectomy.

Methods: We conducted a retrospective study on patients subjected to trabeculectomy with at least a 6-month follow-up. Ophthalmologic examination and spectral-domain optical coherence tomography (SD-OCT) were analyzed. Eyes with previous surgical or laser treatments or other pathologies (other than glaucoma and ERM) were excluded.
\end{abstract}

Results: A total of 50 eyes (40 patients) were included in this study. The mean follow-up time after surgery was 27.8 months. After surgery, 9 eyes $(18 \%)$ had preretinal macular fibrosis and 19 eyes $(38 \%)$ had cellophane macular reflex. Of the 16 eyes with a preoperative macular SD-OCT, $3(18.8 \%)$ developed ERM and 4 $(25 \%)$ progressed from cellophane macular reflex to preretinal macular fibrosis, after surgery. The ERM frequency did not differ significantly between eyes subjected to trabeculectomy with or without the use of antimetabolites $(P=0.08)$, or between eyes subjected to simple or combined surgery (phacotrabeculectomy) $(P=0.09)$.

Conclusion: Trabeculectomy may predispose one to the appearance and progression of ERM. Further studies are needed to clarify this intriguing relation.

Key Words: 5-fluoracyl, cellophane macular reflex, epiretinal membrane, mitomycin-C, preretinal macular fibrosis, phacotrabeculectomy, spectral domain-OCT, trabeculectomy

( J Glaucoma 2016;25:e576-e580)

Ep piretinal membrane (ERM), first described by Iwanoff in $1865,{ }^{1}$ is a vitreomacular interface disorder that can lead to incapacitant metamorphopsia and decreased visual acuity. ERM formation, although still incompletely understood, may result from the proliferation of glial cells, retinal epithelial cells, fibrous astrocytes, fibrocytes, and myofibroblasts after migration through focal defects in the internal limiting membrane, resulting in a thin hiperreflective layer in the vitreomacular interface. ${ }^{2-7}$ The initial form, without foveal alteration, is called cellophane macular reflex (CMR) because of its biomicroscopic appearance and is usually asymptomatic. ${ }^{2,3,6-9}$ In more advanced cases, the ERM contracts, inducing retina traction with

Received for publication December 2, 2014; accepted August 16, 2015. From the *Department of Ophthalmology, Central Lisbon Hospital Center; $†$ Institute of Pharmacology and Neurosciences, Faculty of Medicine, University of Lisbon; and \$NOVA Medical School, Universidade NOVA de Lisboa, Lisbon, Portugal.

Disclosure: The authors declare no conflict of interest.

Reprints: Luisa Vieira, MD, José António Serrano St., Lisbon 1150199, Portugal (e-mail: luisa5vieira@gmail.com).

Copyright (C) 2015 Wolters Kluwer Health, Inc. All rights reserved.

DOI: 10.1097/IJG.0000000000000326 consequent retinal folds and edema, which is known as preretinal macular fibrosis (PMF). ${ }^{2,3,6,7,9}$

Most cases are termed idiopathic and are related to increasing age; however, it has been associated with vascular retinal disorders, ocular trauma and inflammation, high myopia, cataract or retinal detachment surgeries, cryopexy, and laser procedures. ${ }^{3,5-13}$

Trabeculectomy, introduced by Cairns in $1968,{ }^{14}$ aims to reduce the intraocular pressure, when medical control is not possible, to prevent long-term optic nerve damage and maintain the quality of life. After modifications and refinements, this technique is still the standard surgical procedure of glaucoma. Vision loss reported after this procedure is due to cataract development or progression, cystoid macular edema, hypotony, and its potential sequelae and wipe-out syndrome. ${ }^{15,16}$ To our knowledge, the prevalence of ERM after trabeculectomy is not reported in the literature.

Optical coherence tomography (OCT) is a noninvasive, noncontact, transpupillary imaging technology that provides high-resolution cross-sectional images of the retina. With advancements since it was described in 1991, ${ }^{17}$ it is now extensively applied to ocular pathologies ${ }^{18-20}$ and may be more sensitive than biomicroscopy to detect ERM, especially when media opacities are present. $^{2}$

The purpose of our study was to determine the ERM frequency in eyes subjected to trabeculectomy using to OCT.

\section{METHODS}

\section{Participant Selection}

A retrospective case series study was carried out on glaucoma patients subjected to trabeculectomy or phacotrabeculectomy, in the Glaucoma Department of Central Lisbon Hospital Center. All eyes with at least a 6-month follow-up, subjected to surgery between January 2009 and August 2013, were included. Of the 492 eyes subjected to trabeculectomy between these dates, 154 eyes had macular SD-OCT scans after glaucoma surgery. One hundred four eyes were excluded (remaining 50 eyes). The exclusion criteria were refractive error outside the range of $\pm 4 \mathrm{D}$, with previous or subsequent surgical or laser treatments or vascular retinal disorders, retinal detachment, ocular trauma and inflammation, and secondary glaucoma.

\section{Procedures}

Clinical data were assessed, and included the age, the sex, the type of glaucoma, the type of surgery, the use of antimetabolites, and ophthalmologic examination. The age was assessed at the time of postoperative SD-OCT. The follow-up time was measured in months between the surgery date and the last SD-OCT date. 
The existence of ERM (CMR or PMF) was assessed by SD-OCT (Heidelberg Engineering, Heidelberg, Germany). A fast macular-thickness map, centered on the fovea and composed of 25 horizontal scans with $6.1 \mathrm{~mm}$ length separated by $250 \mu \mathrm{m}$, was obtained in 42 cases. In 8 cases, a posterior pole scan program composed of 61 scans in a central 20-degree area was acquired. In the later cases, only alterations in the $6.1 \mathrm{~mm}$ length and $6 \mathrm{~mm}$ in the height were considered. CMR was defined as OCT thickening of the vitreoretinal interface without the loss of foveal depression and without extrafoveal architecture alterations. PMF was defined as OCT thickening of the vitreoretinal interface with loss of foveal depression, possibly associated with the intraretinal fluid as described by Milani et al, ${ }^{2}$ and/ or extrafoveal architecture alterations due to ERM traction. SD-OCT was evaluated off-line by 2 blinded experienced observers for the clinical features of the experimental participants. The presence of ERM in eyes subjected to trabeculectomy with or without the use of antimetabolites and eyes subjected to simple or combined surgery (phacotrabeculectomy) were compared.

\section{Statistical Analysis}

Statistical analysis was conducted using the IBM SPSS Statistics for Windows, version 20.0., IBM Corp., Armonk, NY. The means and SDs were calculated. Continuous and categorical variables were compared using the MannWhitney or the $\chi^{2}$ tests, respectively. Two-tailed $P$-values $<0.05$ were considered to be statistically significant.

\section{RESULTS}

A total of 40 patients (50 eyes), 15 female and 25 male, with a mean age of $64.9 \pm 13.2$ years were included in this study. Forty-three eyes had primary open-angle glaucoma and 7 had pseudoexfoliation syndrome. The mean followup time was $27.5 \pm 17.5$ months. Antimetabolites were used in 33 eyes ( 11 eyes with mitomycin-C and 22 eyes with 5 -fluoracyl). Thirty-five eyes were subjected to trabeculectomy and 15 to phacotrabeculectomy (Table 1).

After surgery, 9 eyes $(18 \%)$ had PMF and 19 eyes $(38 \%)$ had CMR. Of the 16 eyes with preoperative SDOCT, $3(18.8 \%)$ developed ERM (Fig. 1) and $4(25 \%)$ progressed from CMR to PMF (Fig. 2). Of the 9 eyes having PMF, 5 (10\% of the total eyes) underwent vitreoretinal surgery to specifically treat ERM. The ERM frequency did not differ significantly between eyes subjected to trabeculectomy with ( 21 eyes; $63.6 \%$ ) or without ( 7 eyes; $41.2 \%)$ the use of antimetabolites $(P=0.08)$, or between eyes subjected to simple (23 eyes; $65.7 \%)$ or combined surgery $(6$ eyes; $40 \%)(P=0.09)$ (Tables 2,3$)$.

\section{TABLE 1. Experimental Group Features}

\begin{tabular}{lc}
\hline Features & Number \\
\hline N (eyes/patients) & $50 / 40$ \\
Sex ( $(\widehat{\sigma})$ & $15 / 25$ \\
Age $(y)$ & $64.9 \pm 13.2$ \\
With antimetabolites/without antimetabolites (eyes) & $33 / 17$ \\
Trabeculectomy/phacotrabeculectomy (eyes) & $35 / 15$ \\
Follow-up time (mo) & $27.5 \pm 17.5$ \\
\hline
\end{tabular}

Regarding the SD-OCT evaluation, there was an agreement between the 2 observers in all cases.

\section{DISCUSSION}

The ERM prevalence based on biomicroscopy findings varies among the populations between $2.2 \%$ and $39 \%, 3,6,7,9,10,13,21$ with considerable racial and ethnic variations. This disparity may be due to the variability in methods and protocols for grading and defining ERM used in the different studies. Also, on the basis of OCT, Duan et $\mathrm{al}^{8}$ reported an ERM rate of $3.4 \%$ and Milani et $\mathrm{al}^{2}$ described a rate of $26 \%$, although these authors included only eyes of patients who were about to undergo cataract extraction. Our study was based on the detection of ERM using SD-OCT, similar to that described by Milani et al. ${ }^{2}$ Nevertheless, the CRM and the PMF frequency detected in our study ( $38 \%$ and $18 \%$, respectively) was higher than the published data after cataract surgery.

Concerning the ERM frequency with or without the use of antimetabolites, it did not differ between the groups. Although mitomycin- $\mathrm{C}$ and 5-fluoracyl are known to be effective in limiting the fibroblast activity, topical administration does not achieve the vitreous and the retina, ${ }^{22}$ and so may not influence the incidence of ERM.

Even though phacoemulsification is a known risk factor for the appearance of secondary ERM,,$^{3,10,23}$ in our study, phacotrabeculectomy did not contribute significantly to a higher ERM frequency compared with trabeculectomy alone. Similarly, the reported ERM frequency in our study was higher than the published prevalence after cataract surgery $(13.9 \%$ to $28 \%))^{3,10,23}$ The authors hypothesize that a higher and sustained intraocular inflammation for a longer time ${ }^{24}$ and the mechanical forces induced on the vitreoretinal interface after rapid intraocular pressure variations after trabeculectomy, compared with phacoemulsification, may be a possible explanation for this finding.

After evaluating the SD-OCT preoperatively and postoperatively, $18.8 \%$ of the patients developed ERM and $25 \%$ progressed from cellophane reflex to preretinal fibrosis, suggesting that trabeculectomy can induce not only the appearance but also the progression of ERM. The Blue Mountains Eye study ${ }^{5}$ reported an incidence of ERM after 5 years of $5.3 \%$ and a progression of CRM to PMF of $9.3 \%$, and Fong et $\mathrm{al}^{23}$ showed an incidence of ERM after 3 years of $11.2 \%$ and progression from CMR to PMF of $13.5 \%$, which were lower than what we obtained. We found that the ERM was clinically significant in at least $10 \%$ of the eyes, as they met the criteria to be subjected to vitreoretinal surgery.

The authors believe that these findings can lead to important modifications in clinical practice. When patients already have CMR or PMF, but without the indications for surgery, we suggest patient counseling for a possible second intervention. The use of other types of surgeries in these patients, such us deep sclerectomy, with less manipulation of the globe, needs to be evaluated in further studies.

This consecutive case series study has several limitations that include a small sample size (which may affect subgroups' statistical analysis), the lack of a standardized treatment approach, the absence of a control group, and retrospective data collection. Also, the selection criteria used by the authors was to include patients with postoperative macular SD-OCT, and so it is possible that these tests have been requested for a suspicion of macular disease. Nevertheless, we know that these patients still require 
A

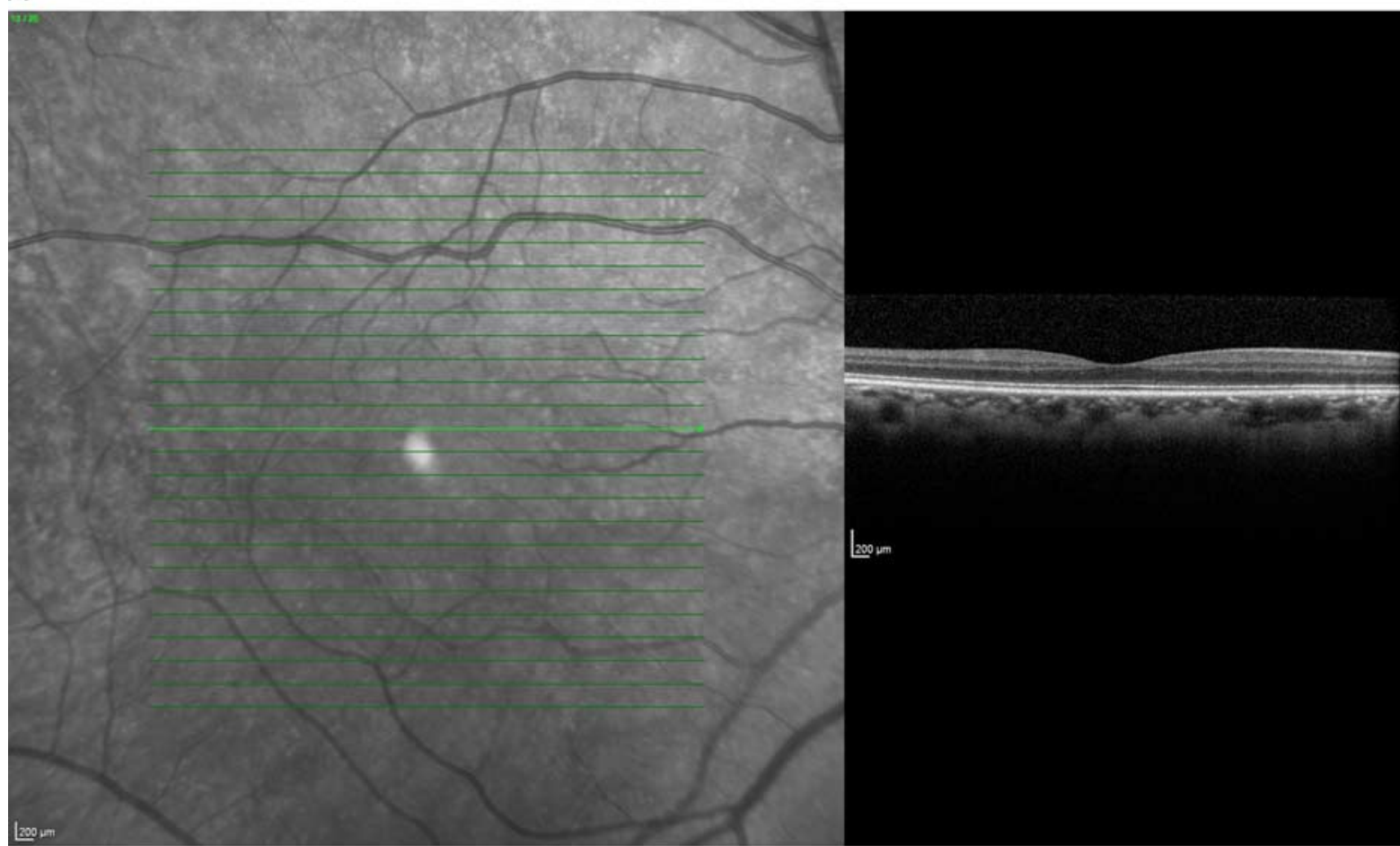

$\mathrm{B}$

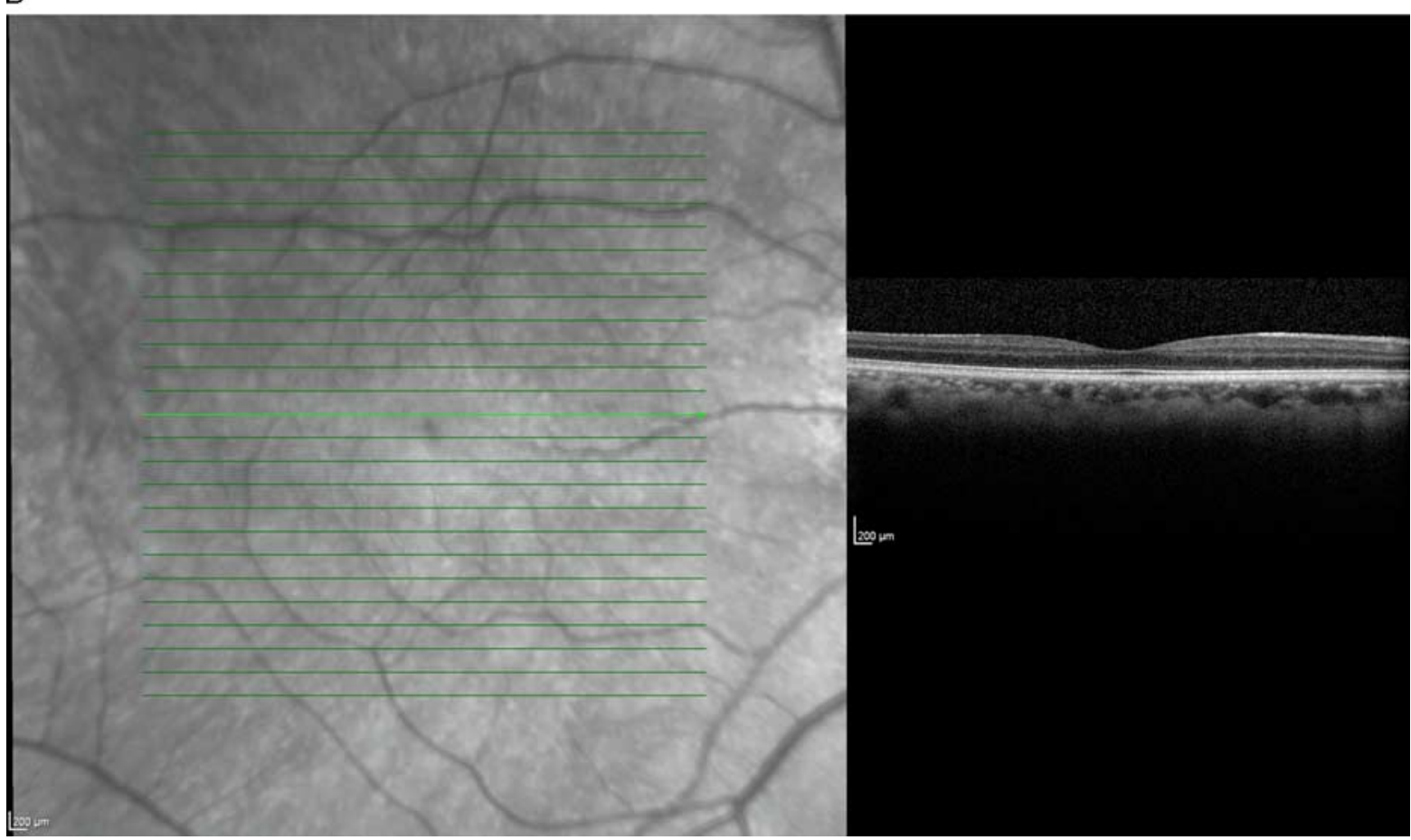

FIGURE 1. Spectral-domain optical coherence tomography (SD-OCT) of a patient who developed cellophane macular reflex after surgery: preoperatively (A); postoperatively (B).

SD-OCT peripapillary scans after surgery, and in the authors' institution, as per the protocol since 2011, macular SD-OCT scans are performed as an add-on to all patients who have an SD-OCT papillary scan, regardless of the reason for the examination underlying the requested test. In our series, only 4 of 50 eyes had postoperative scans performed before 2011. The low number of patients who had SD-OCT preoperatively is explained by the fact that the majority of the patients were subjected to surgery in 2009 and 2010, and so macular SD-OCT may have been 
A

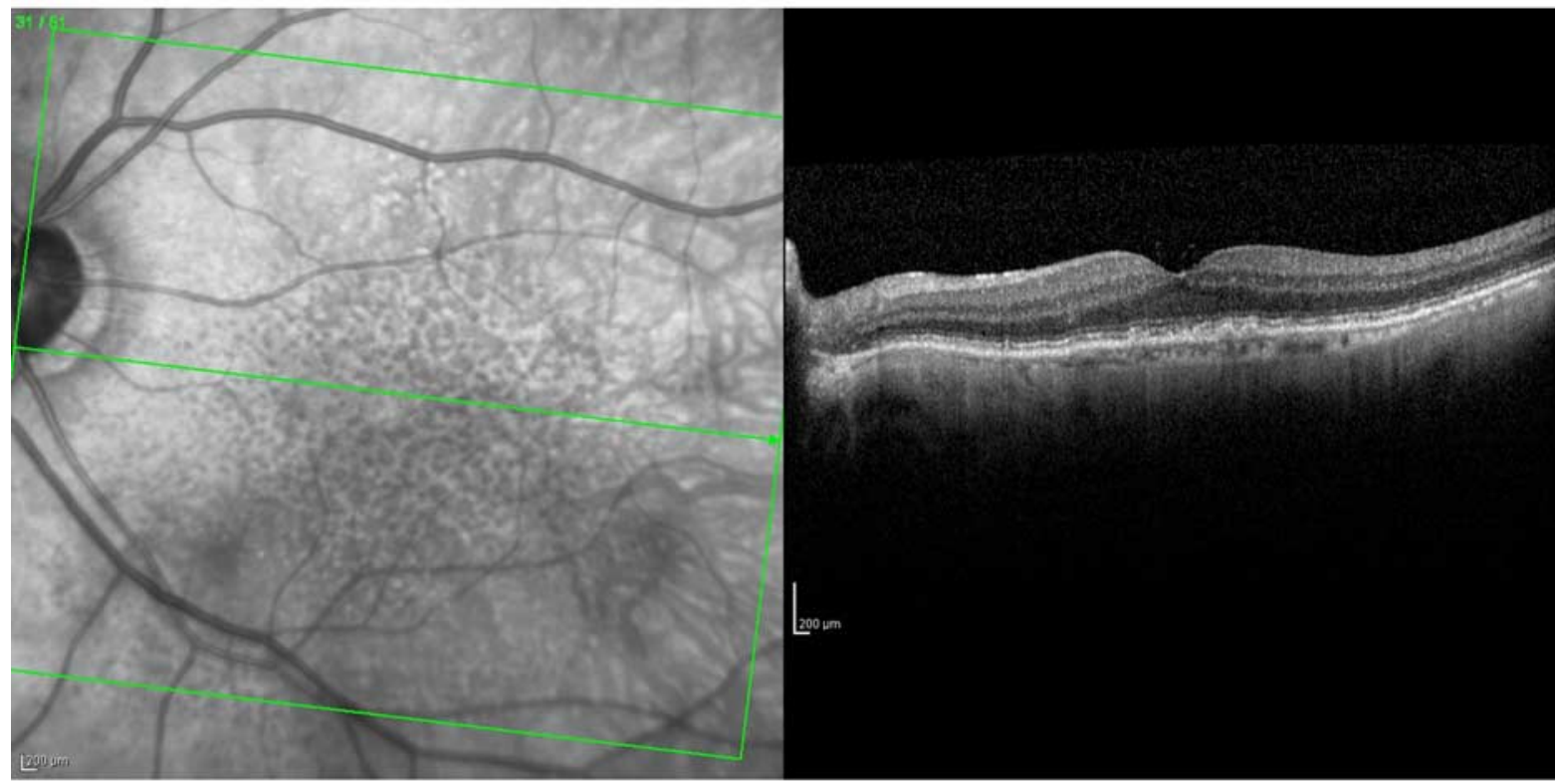

B

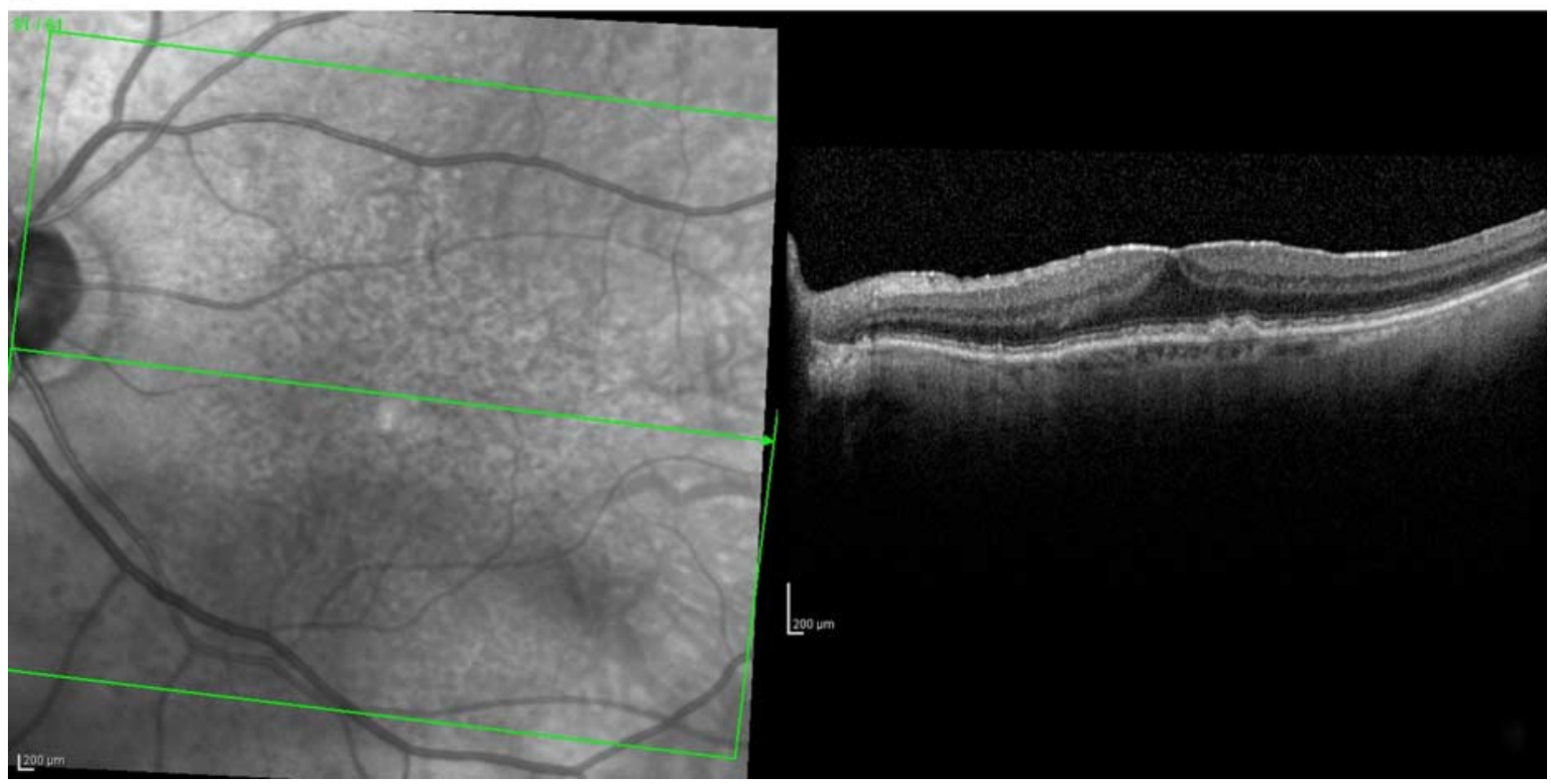

FIGURE 2. Spectral-domain optical coherence tomography (SD-OCT) of a patient who developed preretinal macular fibrosis after surgery: preoperatively (A); postoperatively (B).

requested only if there was a clinical suspicion of macular disorder. Actually, it would be interesting to carry out a prospective study and evaluate the incidence of ERM after this type of glaucoma surgery. Another potential limitation is that CMR diagnosis on SD-OCT may raise some doubts.

TABLE 2. The Frequency of Epiretinal Membrane (ERM) With or Without the Use of Antimetabolites

\begin{tabular}{lccc}
\hline & $\begin{array}{c}\text { With } \\
\text { Antimetabolites }\end{array}$ & $\begin{array}{c}\text { Without } \\
\text { Antimetabolites }\end{array}$ & $\boldsymbol{\chi}^{\mathbf{2}}(\boldsymbol{P})$ \\
\hline ERM (n/total) & $21 / 33$ & $7 / 17$ & 0.08 \\
\hline
\end{tabular}

However, in our study, there was an agreement between the observers in all cases (CMR cases included).

Despite such limitations, to our knowledge, this is the first study reporting the ERM appearance after trabeculectomy.

Our results suggest that trabeculectomy may predispose one to the appearance and progression of ERM.

TABLE 3. The Frequency of Epiretinal Membrane (ERM) in Trabeculectomy and Phacotrabeculectomy Groups

\begin{tabular}{lccc}
\hline & Trabeculectomy & Phacotrabeculectomy & $\boldsymbol{\chi}^{\mathbf{2}(\boldsymbol{P})}$ \\
\hline ERM (n/total) & $23 / 35$ & $6 / 15$ & 0.09 \\
\hline
\end{tabular}


Clinicians should evaluate macular alterations cautiously during the preoperative evaluation of eyes that are going to be subjected to trabeculectomy.

\section{REFERENCES}

1. Iwanoff A. Beitrage zur normalen und pathologischen anatomy des auges [Contributions to the normal and pathological anatomy of the eye]. Graefes Arch Clin Exp Ophthalmol. 1865;11:135-170.

2. Milani P, Raimondi G, Morale D, et al. Biomicroscopy versus optical coherence tomography screening of epiretinal membranes in patients undergoing cataract surgery. Retina. 2012; 32:897-904.

3. McCarty DJ, Mukesh BN, Chikani V, et al. Prevalence and associations of epiretinal membranes in the visual impairment project. Am J Ophthalmol. 2005;140:288-294.

4. Mori K, Gehlbach PL, Sano A, et al. Comparison of epiretinal membranes of differing pathogenesis using optical coherence tomography. Retina. 2004;24:57-62.

5. Fraser-Bell S, Guzowski M, Rochtchina E, et al. Five-year cumulative incidence and progression of epiretinal membranes: the Blue Mountains Eye Study. Ophthalmology. 2003;110:34 40.

6. Fraser-Bell S, Ying-Lai M, Klein R, et al. Los Angeles Latino Eye Study. Prevalence and associations of epiretinal membranes in latinos: the Los Angeles Latino Eye Study. Invest Ophthalmol Vis Sci. 2004;45:1732-1736.

7. Aung KZ, Makeyeva G, Adams MK, et al. The prevalence and risk factors of epiretinal membranes: the Melbourne Collaborative Cohort Study. Retina. 2013;33:1026-1034.

8. Duan XR, Liang YB, Friedman DS, et al. Prevalence and associations of epiretinal membranes in a rural Chinese adult. Population: the Handan Eye Study. Invest Ophthalmol Vis Sci. 2009;50:2018-2023.

9. $\mathrm{Ng} \mathrm{CH}$, Cheung N, Wang JJ, et al. Prevalence and risk factors for piretinal membranes in a multi-ethnic United States population. Ophthalmology. 2011;118:694-699.

10. Mitchell P, Smith W, Chey T, et al. Prevalence and associations of epiretinal membranes. The Blue Mountains Eye Study, Australia. Ophthalmology. 1997;104:1033-1040.
11. Appiah AP, Hirose T. Secondary causes of premacular fibrosis. Ophthalmology. 1989;96:389-392.

12. Kraushar MF, Morse $\mathrm{PH}$. The relationship between retina surgery and preretinal macular fibrosis. Ophthalmic Surg. 1988; 19:843-848.

13. Klein R, Klein BE, Wang Q, et al. The epidemiology of epiretinal membranes. Trans Am Ophthalmol Soc. 1994;92: 403-425.

14. Cairns JE. Trabeculectomy. Preliminary report of a new method. Am J Ophthalmol. 1968;66:673-679.

15. El Sayyad F, Helal M, El-Kholify H, et al. Nonpenetrating deep sclerectomy versus trabeculectomy in bilateral primary open-angle glaucoma. Ophthalmology. 2000;107:1671-1674.

16. Francis BA, Hong B, Winarko J, et al. Vision loss and recovery after trabeculectomy: risk and associated risk factors. Arch Ophthalmol. 2011;129:1011-1017.

17. Huang D, Swanson EA, Lin CP, et al. Optical coherence tomography. Science. 1991;254:1178-1181.

18. Wolf S, Wolf-Schnurrbusch U. Spectral-domain optical coherence tomography use in macular diseases: a review. Ophthalmologica. 2010;224:333-340.

19. Adhi M, Duker JS. Optical coherence tomography - current and future applications. Curr Opin Ophthalmol. 2013;24: 213-221.

20. BusselII, Wollstein G, Schuman JS. OCT for glaucoma diagnosis, screening and detection of glaucoma progression. Br J Ophthalmol. 2014;98(suppl 2):ii15-ii19.

21. You $\mathrm{Q}, \mathrm{Xu} \mathrm{L}$, Jonas JB. Prevalence and associations of epiretinal membranes in adult Chinese: the Beijing eye study. Eye (Lond). 2008;22:874-879.

22. Sarraf D, Eezzuduemhoi RD, Cheng Q, et al. Aqueous and vitreous concentration of mitomycin $\mathrm{C}$ by topical administration after glaucoma filtration surgery in rabbits. Ophthalmology. 1993;100:1574-1579.

23. Fong CS, Mitchell $\mathrm{P}$, Rochtchina $\mathrm{E}$, et al. Incidence and progression of epiretinal membranes in eyes after cataract surgery. Am J Ophthalmol. 2013;156:312.e1-318.e1.

24. Siriwardena D, Kotecha A, Minassian D, et al. Anterior chamber flare after trabeculectomy and after phacoemulsification. Br J Ophthalmol. 2000;84:1056-1057. 